\title{
BMJ Open Does 'Time Together' increase quality of interaction and decrease stress? A study protocol of a multisite nursing intervention in psychiatric inpatient care, using a mixed method approach
}

Jenny Molin, ${ }^{1}$ Britt-Marie Lindgren, ${ }^{1}$ Ulla Hällgren Graneheim, ${ }^{1}$ Anders Ringnér ${ }^{1,2}$

To cite: Molin J, Lindgren B-M, Graneheim UH, et al. Does 'Time Together' increase quality of interaction and decrease stress? A study protocol of a multisite nursing intervention in psychiatric inpatient care, using a mixed method approach. BMJ Open 2017;7:e015677. doi:10.1136/ bmjopen-2016-015677

- Prepublication history for this paper is available online. To view these files please visit the journal online (http://dx.doi. org/10.1136/bmjopen-2016015677).

Received 3 January 2017 Accepted 30 June 2017

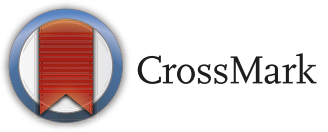

${ }^{1}$ Department of Nursing, Umeå University, Umeå, Sweden

${ }^{2}$ Department of Paediatrics, Umeå University Hospital, Umeå, Sweden

Correspondence to Dr Anders Ringnér; anders.ringner@umu.se

\section{ABSTRACT}

Introduction Despite the long-known significance of the nurse-patient relationship, research in psychiatric inpatient care still reports unfulfilled expectations of, and difficulties in, interactions and relationships between patients and staff. Interventions that create structures to allow quality interactions between patients and staff are needed to solve these problems. The aim of this project is to test effects of the nursing intervention Time Together and to evaluate the intervention process.

Methods and analysis This is a multisite study with a single-system experimental design using frequent measures. The primary outcomes are quality interactions for patients and perceived stress for staff. Secondary outcomes are levels of symptoms of anxiety and depression for patients and stress of conscience for staff. A process evaluation is performed to describe contextual factors and experiences. Data are collected using questionnaires, participant observations and semistructured interviews. For analysis of quantitative data, both visual and statistical methods will be used. Qualitative data will be analysed using qualitative content analysis.

Ethics and dissemination Ethical approval was granted by the Ethical Review Board in the region (Dnr 2016/33931). The findings will contribute to the development of nursing interventions in general, but more specifically to the development of the intervention. This is relevant both nationally and internationally as similar interventions are needed but sparse. The findings will be disseminated through conference presentations and peer-reviewed publications.

Trial registration number NCT02981563

\section{INTRODUCTION}

It is well known that the nurse-patient relationship is the core of mental health nursing. ${ }^{1-4}$ To create such relationships, it is essential that interactions contain emotional communication, ${ }^{15}$ and to convey such care in their responses, staff should reach out to the patient as a first step in sharing that person's experiences. ${ }^{6}$ Barker and
Strengths and limitations of this study

- Multisite study performed at three different wards.

- Process evaluation integrated in the study.

- Relatively small scale could pose a threat to generalisation.

- A single-system experimental design enables us to closely follow the outcome measures.

Buchanan-Barker ${ }^{2} \quad 6 \quad$ conceptualised such human processes as engagement and later renamed it bridging.

Despite the long-known significance of the nurse-patient relationship, research in psychiatric inpatient care still reports unfulfilled expectations of, and difficulties in, interactions and relationships between staff and patients. Patients express a need for human relations with staff and for something to do, while staff say that they have ideals about providing good care, but their time spent with patients is limited by organisational chaos. ${ }^{7-12}$ Patients and staff also seem to share negative experiences of contextual factors such as the environment, the lack of structure and activities, the disorganisation and the substantial power relationships. ${ }^{9} 13-15$ Together, this leads to counterproductive care, experiences of stigma among patients ${ }^{14} 16$ and signs of moral distress among staff. ${ }^{15} 17$

In response to the challenges outlined above, we need to proceed from descriptions to action. Several researchers raise nurses' need for time to devote to their patients. ${ }^{11} 121418$ Similar ideas are discussed by Cleary et $a l^{19}$ and Polacek et $a l^{20}$ who suggest that the focus should be on creating conditions that enable engagement and the development of therapeutic interaction skills with a 'human touch'. ${ }^{21}$ It has also been suggested that being given the responsibility 
and the ability to work in line with their ideals and engage with their patients could reduce moral distress among staff and improve both the quality of care and the chances of recovery for patients. $.^{15} 1722-28$ Together this indicates that nursing interventions focusing on quality interactions could help to solve the described shortcomings in care. However, in a review, Mullen ${ }^{29}$ reported that such interventions, for example, planned dialogues and psychoeducation, are complex and difficult to implement in psychiatric inpatient care because of the demanding and chaotic context. This seems to still be the case, as such interventions are sparsely described in the scientific literature, which implies a need for interventions that create structures to allow quality interactions between staff and patients.

As a grass-roots initiative, one intervention aimed to create structures for quality interactions between staff and patients was developed in the UK in the early 2000s. ${ }^{30}$ There are some descriptions of the practical structures of protected engagement time (PET) in the literature, ${ }^{30-34}$ and evaluations of PET are ongoing in the UK. ${ }^{33}$ The theoretical framework of PET is vaguely described in the early literature. However, according to Nolan $e t a l,{ }^{33}$ the intervention is rooted in practice with a starting point in the staffs' situation as PET was developed on the basis of Karasek and Theorell's theory on job strain, ${ }^{35}$ but with the nurse-patient relationship in the centre. The structure of PET requires that staff, for a fixed time during the day, dedicate their time exclusively to interacting with the patients. Other more administrative duties, visits and meetings are organised to be performed at other times during the day. ${ }^{33}$ Through this, opportunities for interaction are both created and protected.

During the development of our intervention, Barker and Buchanan-Barker's theoretical framework ${ }^{2}$ was added to highlight the centrality of needs of the patients and engagement among staff. There are obvious parallels between the content of PET and the philosophy of Barker and Buchanan-Barker. ${ }^{2}$ They share an emphasis on engagement; both describe levels of engagement in supporting patients in psychiatric inpatient care, but they also take a pragmatic approach to interventions that should be shaped by prevailing requirements and available resources. ${ }^{36}$

Our intervention builds on PET, but expands the theoretical framework by adding Barker and Buchanan-Barker's theory. ${ }^{2}$ To emphasise this theoretical expansion and to adapt the intervention to the Swedish context and language, the intervention described in this project is named Time Together (TT).

\section{STUDY AIMS}

The overall aim of this project is to test the effects of the nursing intervention TT and to evaluate the intervention process. The main research questions are:

- Does TT influence the quality of interactions between staff and patients?
- Does TT influence patients' levels of anxiety and depressive symptoms?

- Does TT influence staffs' levels of perceived stress and levels of stress of conscience?

- Does TT influence the prevalence of coercive measures, mean length of hospital stay and the use of PRN (as needed) medication?

Because this will be the first use of TT in Swedish psychiatric inpatient care there is also a need to evaluate the process of introducing it as a nursing intervention. Through this process evaluation, we aim to answer questions such as:

- How do staff and patients describe their experiences of the intervention and how do contextual factors influence the effects of the intervention?

- What are the relationships between the outcome variables and the degree of compliance with the intended intervention?

-What problems are there with recruitment and dropouts?

\section{METHODS}

This is a multisite nursing intervention project using a single-system experimental design (SSED). ${ }^{37}$

SSED studies focus primarily on changes in one system and not differences between systems. Each system works as its own control and therefore a smaller number of systems are required than in other experimental models. ${ }^{38}$ The process evaluation is performed in parallel to describe experiences and contextual factors of importance.

In this project, the evaluation of TT will consist of two phases (A and B) and follow-up, in line with the SSED. In the A phase, where the baseline is established, outcomes will be measured once every seventh day (weekends not included) for approximately 5 weeks. In B phase, TT will be introduced on the wards and outcomes will be measured once every seventh day (weekends not included) for 3 months. The follow-up will take place 6 months after the B phase. Both patients and staff will complete questionnaires and participate in interviews to assess whether the possible effects of TT are sustainable.

The project will be conducted from January 2017 to May 2018 at three psychiatric clinics located at three hospitals in two county councils in the north of Sweden. One psychiatric inpatient care ward at each clinic will participate. The samples in this study are then the three wards. As single-system designs aim at detecting changes within each system and not comparing these to each other, sample sizes in this tradition are comparably small and no sample size calculations are made. ${ }^{38}$ Each ward constitutes one system and data for each system will consist of aggregated measurements from admitted patients and staff working on each ward. ${ }^{37} 39$ This implies that that the total number of individuals is largely dependent on (1) the number of patients admitted to the ward at each point of measurement and (2) the number of staff members working at the ward. 


\section{Description of TT}

A description similar to that of Nolan et $a l^{33}$ in combination with Barker and Buchanan-Barker's description of levels of engagement ${ }^{2}$ will be used as the basis for the introduction of TT. The basic model for the intervention will therefore be:

- Regular times for TT will be established on MondaysFridays, for a total of 5 hours.

- Registered nurses (RNs) and enrolled nurses (ENs) will be engaged in joint activities with the patients during TT.

- Engagement should involve interactions in either one-on-one sessions or group sessions including joint activities chosen by the patients.

- During TT, only one or two of the ward staff will manage the administrative ward duties. All other members of staff will engage with the patients.

- During TT, the ward will be closed to visitors and professionals from outside the ward.

For the intervention to be feasible, TT will be tailored to local circumstances at each ward with the points above as a basic guide. ${ }^{40}$

\section{Setting}

The patients on the participating wards, admitted voluntarily or involuntarily, suffer from different kinds of mental ill health or substance-related and addictive disorders. In general, the wards have rules and routines, locked doors, fixed times for meals, smoking breaks and opportunities to walk outdoors. Nursing interventions such as joint activities, planned dialogues and psychoeducation are usually rare, and medical treatment is the norm instead. Those working on the wards are ENs in mental health, RNs, some of whom have specialist training in mental health nursing, a ward manager, residents and consultant psychiatrists. Other professions can be consulted when necessary. For a description of the intervention wards, please refer to table 1 .

\section{Participants}

All patients admitted to the wards during phase A, phase $\mathrm{B}$ and the follow-up of the intervention will be informed about the ongoing project by a research assistant. During phase A, all patients admitted to the wards will be invited to participate in the study by completing questionnaires. During phase B, the patients who participated in at least one TT session will be invited to participate through

\begin{tabular}{llll}
\hline Table 1 & \multicolumn{2}{l}{ Description of the three intervention wards } \\
\hline $\begin{array}{l}\text { Ward } \\
\text { no }\end{array}$ & Subspecialty & $\begin{array}{l}\text { No of } \\
\text { beds }\end{array}$ & $\begin{array}{l}\text { Total no of } \\
\text { eligible staff }\end{array}$ \\
\hline 1 & $\begin{array}{l}\text { Substance and addictive } \\
\text { syndromes }\end{array}$ & 12 & 17 \\
2 & $\begin{array}{l}\text { Substance and addictive } \\
\text { syndromes }\end{array}$ & 12 & 25 \\
3 & Acute mental health & 13 & 19 \\
\hline
\end{tabular}

questionnaires, semistructured interviews and participant observations.

Inclusion criteria for patients: 18 years or older, admitted to the wards during phase A, phase B and/or follow-up. Experience of at least one TT session is required.

Exclusion criteria for patients: not fluent enough in the Swedish language to complete questionnaires and participate in interviews.

The researchers will invite all staff working on the wards during phase A, phase B and follow-up of the intervention to participate in the evaluation of TT.

Inclusion criteria for staff: staff employed at the ward during phase A, phase B and/or follow-up.

\section{Data collection}

Data will be collected through questionnaires and ward registers. In the evaluation of the process, semistructured interviews and participant observations will be conducted by the researchers and logbooks will be kept by the research assistants at each ward.

\section{Questionnaires}

The primary outcome measure for patients will be the quality of interactions with members of staff, based on the results of previous studies ${ }^{14}$ that showed that, according to patients in psychiatric inpatient care, the quality of interactions influences everyday life in psychiatric inpatient care. Secondary outcome measures will be levels of anxiety and depressive symptoms. For staff, the primary outcome measure will be perceived stress, based on the results of previous studies ${ }^{151726}$ that showed that staff in interprofessional teams in psychiatric inpatient care moved from ideals to resignation because of organisational and structural obstacles. Secondary outcome measures will be stress of conscience, satisfaction of interactions with patients and quality of care. Demographic data will be collected for all participants.

\section{Patient-related questionnaires}

The Caring Professional Scale (CPS) ${ }^{42}$ will be used to measure the quality of the interactions. CPS consists of 14 items answered on a five-point Likert scale. Validity and reliability has been reported as satisfactory (Cronbach's alpha nurses 0.97). ${ }^{42}$

The widely used and well-tested Visual Analogue Scale (VAS) ${ }^{43}$ will be used to measure satisfaction with the interactions. The scale will be $100 \mathrm{~mm}$ in length, ranging from 'very unsatisfactory' to 'very satisfactory'.

The self-assessment scale The Hospital Anxiety and Depression Scale (HAD) ${ }^{44}$ will be used to measure anxiety and depressive symptoms. All items are scored on a fourpoint scale. HAD appears to be reliable and valid and is shown to be sensitive to changes in response to psychosocial interventions. ${ }^{445}$ The Swedish version was tested and shown to have satisfactory validity and reliability (Cronbach's alpha 0.90). ${ }^{46}$ 


\section{Staff related questionnaires}

The Perceived Stress Scale (PSS) ${ }^{47}$ will be used to measure stress among staff. PSS consists of 10 items answered on a five-point Likert scale. The Swedish version of the 10-item PSS has proved to have satisfactory validity and reliability (Cronbach's alpha 0.84). ${ }^{48}$

The Stress of Conscience Questionnaire (SCQ) ${ }^{49}$ will be used to measure the frequencies of stressful situations and the degree to which these lead to stress of conscience among staff. SCQ consists of nine items in two parts. The first part uses a six-point scale ranging from never $(0)$ to everyday (5) for each of the nine items and the second part uses a $100 \mathrm{~mm}$ VAS ranging from 'No, not at all' (0) to 'Yes, it gives me a very troubled conscience' (5) for those same items. Previous studies have reported satisfactory validity and reliability (Cronbach's alpha 0.83 for the total SCQ). ${ }^{50}$

The VAS $^{43}$ will be used to measure staff satisfaction with their interactions with patients. The scale will be $100 \mathrm{~mm}$ in length, ranging from 'very unsatisfactory' to 'very satisfactory'.

The Quality in Psychiatric Care-Inpatient Staff (QPCIPS) ${ }^{51}$ questionnaire will be used to measure quality of care. This instrument is part of the QPG family of instruments that originate from the QPC study. ${ }^{51}$ Psychometric tests have been conducted internationally and are ongoing nationally. No results have yet been published.

\section{Data from ward registers}

Data regarding PRN medication, hospital stays, use of coercion and violent situations will be collected from the participating wards' existing registers.

\section{Semistructured interviews}

Both patients and staff will be individually interviewed by the researchers, using a semistructured format. ${ }^{53}$ During phase B, patients will be interviewed just before or shortly after their discharge from the ward and staff will be interviewed at the end of the phase. The participants will be asked to share their experience of TT's impact on the quality of the interactions. For staff, there will also be questions about TT's impact on their daily work and organisational issues that facilitate and complicates TT. For this process evaluation component of the study, the sample will be selected purposively by the researchers. We will strive at describing variations in experience of the intervention. This will approximately require 30 staff members and 30 patients (10 from each ward), however, interviews will be performed until saturation is achieved. $^{54}$

\section{Participant observations}

Participant observations will be used during phase B. This approach, aimed to describe patterns of behaviours among individuals and groups in a particular culture, gives researchers the opportunity to share certain experiences with both patients and staff on the wards. In this project, observations with a focused approach will allow researchers to describe activities and interactions between patients and staff during TT. ${ }^{55}$

$\log$

During phase B, the research assistant at each ward will keep a log recording the number of TT sessions, their duration, the kinds of activities engaged in, and reflections on each session.

\section{Analysis}

Quantitative data

Demographic data for participants will be presented as means and proportions. To evaluate changes during the intervention, both visual and statistical methods will be used to analyse data. ${ }^{56}$ The visual inspection of data in SSED studies is described as a viable and approved method to analyse and compare levels, trends and variability between intervention phases. ${ }^{38}$ For the statistical calculations, percentage of non-overlapping data statistics will be used. This is a calculation of the percentage of treatment data that overlaps with the most extreme data point exhibited at baseline. ${ }^{38}$

\section{Qualitative data}

Both the participant observations and the semistructured interviews will be analysed using qualitative content analysis (QCA). In QCA the focus is on variations in participants' experiences and presenting the results in categories and/or themes. ${ }^{57}$

\section{ETHICS AND DISSEMINATION}

The study has been approved by the heads of the clinical departments of psychiatry involved and the regional Ethical Review Board in Umeå, Sweden (Dnr 2016/339-31). All participants will receive written and verbal information about the aim of the study. They will be informed that participation is voluntary, that they have the right to withdraw without specifying why, and that confidentiality will be assured. Informed consent will be assigned by all participants. The findings will be disseminated through conference presentations and peer-reviewed publications.

\section{DISCUSSION}

Introducing TT is not only an attempt to meet the needs described by patients and staff in psychiatric inpatient care, but also an attempt to come to terms with some of the shortcomings described in the evidence (ie, lack of structure and unmanaged chaos). ${ }^{14} 15$ To our knowledge, similar studies are sparse in the international evidence and no similar projects have been tested or implemented in the context of Swedish psychiatric inpatient care.

The project, including the SSED and the process evaluation, will provide a first description of TT in a Swedish context. It is significant because studies of this type are sparse both nationally and internationally. Research in mental health nursing has a responsibility to take the 
next step since many of the problematic circumstances now are known. The development of interventions is a step in the right direction towards solving some of these problems. ${ }^{58}$

This study is performed at three hospital wards. Even though this may be considered a small sample with a limited generalisability, this study will still assess the effect of TT in this context. Such knowledge will provide a basis for further development and indications of what adjustments needs to be done to better suit psychiatric inpatient care nationally and possibly, even internationally in similar context.

Combining the SSED with a process evaluation has been described as highly valuable. ${ }^{59}$ Collecting both quantitative and qualitative data will offer us opportunities to describe the introduction of TT from different viewpoints, enable us to capture a broad picture of the effects of TT, and enable us to evaluate the trustworthiness and quality of the introduction of the intervention. ${ }^{59}$ This in turn could contribute to the development and dissemination of TT.

According to Craig et $a l^{40}$ a distinct theoretical framework is the basis of sustainable interventions and allows for better evaluations. Barker and Buchanan-Barker's concept of engagement ${ }^{2}$ is added as a theoretical framework for this project. This stance, combined with the introduction of TT to the Swedish context will provide opportunities for further development of the intervention.

The composition of the research team provides a mix of experience in the context and the methods used for evaluation. JM, BML and UHG are experienced mental health nurses and AR and UHG have use SSED, including the quantitative methods used during analysis. All authors are experienced in qualitative methods.

In the first descriptions, the PET intervention is described to have its roots in practice rather than in theory. ${ }^{30}$ This pragmatic approach has been preserved in the design of TT which will be tailored to local circumstances at each participating ward. This has been done in collaboration with staff working on the wards and patients have also been asked about what joint activities they want to be offered during TT. ${ }^{56}$ Such an approach, according to Storm and Edwards, ${ }^{60}$ is fundamental to promote user involvement. However, this could be seen as a study limitation as the intervention then is less standardised. Still, it has been key to introducing a feasible intervention that would be realistic to implement if it is proven to be effective. As described, we see the potential of the intervention because it does not require additional staff or costs, being in line with the wishes of the patients and the ideals of the staff. ${ }^{1415}$

Contributors Study design: JM, AR, B-ML, UHG. Manuscript preparation: JM, AR, BML, UHG. All authors read and approved the final manuscript.

Funding This research is funded by The Swedish National Association for Social and Mental Health, the Kempe Foundation and the Swedish Society of Nursing. The research is independent, however, and the views expressed in this article are solely those of the authors.

Competing interests None declared.
Ethics approval Regional Ethical Review Board in Umeå.

Provenance and peer review Not commissioned; externally peer reviewed.

Open Access This is an Open Access article distributed in accordance with the Creative Commons Attribution Non Commercial (CC BY-NC 4.0) license, which permits others to distribute, remix, adapt, build upon this work non-commercially, and license their derivative works on different terms, provided the original work is properly cited and the use is non-commercial. See: http://creativecommons.org/ licenses/by-nc/4.0/

(c) Article author(s) (or their employer(s) unless otherwise stated in the text of the article) 2017. All rights reserved. No commercial use is permitted unless otherwise expressly granted.

\section{REFERENCES}

1. Altschul AT. Patient-Nurse Interaction: a study of interacton patterns in acute psychiatric wards. Edinburgh: Churchill Livingstone, 1972.

2. Barker P, Buchanan-Barker P. The Tidal Model: a guide for mental health professionals. London: Routledge, 2005.

3. Halldorsdottir $\mathrm{S}$. The dynamics of the nurse-patient relationship: introduction of a synthesized theory from the patient's perspective. Scand J Caring Sci 2008;22:643-52.

4. Peplau HE. Interpersonal relations in nursing: a conceptual frame of reference for psychodynamic nursing. New York 1952.

5. Rogers CR. On becoming a person: a therapist's view on psychotherapy. Boston: Houghton Mifflin, 1978.

6. Barker P, Buchanan-Barker P. Bridging: talking meaningfully about the care of people at risk. Mental Health Practice 2004;8:12-15.

7. Cleary $M$. The realities of mental health nursing in acute inpatient environments. Int J Ment Health Nurs 2004;13:53-60.

8. Goulter N, Kavanagh DJ, Gardner G. What keeps nurses busy in the mental health setting? J Psychiatr Ment Health Nurs 2015;22:449-56.

9. Graneheim UH, Slotte A, Säfsten HM, et al. Contradictions between ideals and reality: Swedish registered nurses' experiences of dialogues with inpatients in psychiatric care. Issues Ment Health Nurs 2014;35:395-402.

10. Lindgren BM, Aminoff C, Hällgren Graneheim U. Features of everyday life in psychiatric inpatient care for self-harming: an observational study of six women. Issues Ment Health Nurs 2015;36:82-8.

11. Stenhouse RC. 'They all said you could come and speak to us': patients' expectations and experiences of help on an acute psychiatric inpatient ward. J Psychiatr Ment Health Nurs 2011;18:74-80.

12. Wood L, Alsawy S. Patient experiences of psychiatric inpatient care: a systematic review of qualitative evidence. Journal of Psychiatric Intensive Care 2016;12:35-43.

13. Delaney KR, Johnson ME. Metasynthesis of research on the role of psychiatric inpatient nurses: what is important to staff? $\mathrm{J} \mathrm{Am}$ Psychiatr Nurses Assoc 2014;20:125-37.

14. Molin J, Graneheim UH, Lindgren BM. Quality of interactions influences everyday life in psychiatric inpatient care--patients perspectives. Int J Qual Stud Health Well-being 2016;11:29897.

15. Molin J, Graneheim UH, Ringnér A, et al. From ideals to resignation - interprofessional teams perspectives on everyday life processes in psychiatric inpatient care. J Psychiatr Ment Health Nurs 2016;23:595-604.

16. Ejneborn Looi GM, Engström Å, Sävenstedt S. A self-destructive care: self-reports of people who experienced coercive measures and their suggestions for alternatives. Issues Ment Health Nurs 2015;36:96-103.

17. Gabrielsson S, Sävenstedt S, Olsson M. Taking personal responsibility: Nurses' and assistant nurses' experiences of good nursing practice in psychiatric inpatient care. Int J Ment Health Nurs 2016;25:434-43.

18. McAndrew S, Chambers M, Nolan F, et al. Measuring the evidence: reviewing the literature of the measurement of therapeutic engagement in acute mental health inpatient wards. Int J Ment Health Nurs 2014;23:212-20.

19. Cleary M, Hunt GE, Horsfall J, et al. Nurse-patient interaction in acute adult inpatient mental health units: a review and synthesis of qualitative studies. Issues Ment Health Nurs 2012;33:66-79.

20. Polacek MJ, Allen DE, Damin-Moss RS, et al. Engagement as an Element of Safe Inpatient Psychiatric Environments. J Am Psychiatr Nurses Assoc 2015;21:181-90.

21. Gunasekara I, Pentland T, Rodgers T, et al. What makes an excellent mental health nurse? A pragmatic inquiry initiated and conducted by 
people with lived experience of service use. Int J Ment Health Nurs 2014;23:101-9.

22. Bowles N, Howard R. The Refocusing Model: A Means of Realising the National Acute Inpatient Strategy. Mental Health Review Journal 2003;8:27-31.

23. Dodds $P$, Bowles N. Dismantling formal observation and refocusing nursing activity in acute inpatient psychiatry: a case study. $J$ Psychiatr Ment Health Nurs 2001;8:183-8.

24. Edwards D, Burnard P. A systematic review of stress and stress management interventions for mental health nurses. $J$ Adv Nurs 2003;42:169-200.

25. Hanrahan NP, Aiken LH, McClaine L, et al. Relationship between psychiatric nurse work environments and nurse burnout in acute care general hospitals. Issues Ment Health Nurs 2010;31:198-207.

26. Tuvesson $\mathrm{H}$, Eklund $\mathrm{M}$, Wann-Hansson $\mathrm{C}$. Perceived stress among nursing staff in psychiatric inpatient care: the influence of perceptions of the ward atmosphere and the psychosocial work environment. Issues Ment Health Nurs 2011;32:441-8.

27. Tuvesson H, Eklund M, Wann-Hansson C. Stress of Conscience among psychiatric nursing staff in relation to environmental and individual factors. Nurs Ethics 2012;19:208-19.

28. Van Bogaert $P$, Wouters $K$, Willems $R$, et al. Work engagement supports nurse workforce stability and quality of care: nursing teamlevel analysis in psychiatric hospitals. J Psychiatr Ment Health Nurs 2013;20:679-86.

29. Mullen A. Mental health nurses establishing psychosocial interventions within acute inpatient settings. Int $J$ Ment Health Nurs 2009;18:83-90.

30. Kent M. My mental health. Mental Health Practice 2005;8:22.

31. Edwards K, Dhoopnarain A, Fellows J, et al. Evaluating protected engagement time in mental health acute care. Nursing Times 2008:28-9.

32. McCrae N. Protected engagement time in mental health inpatient units. Nurs Manag 2014;21:28-31.

33. Nolan FM, Fox C, Cheston R, et al. A feasibility study comparing UK older adult mental health inpatient wards which use protected engagement time with other wards which do not: study protocol. Pilot Feasibility Stud 2016;2:1.

34. Thomson LD, Hamilton R. Attitudes of mental health staff to protected therapeutic time in adult psychiatric wards. J Psychiatr Ment Health Nurs 2012;19:911-5.

35. Karasek R. Theorell T. Healthy work: stress, productivity, and the reconstruction of working life. New York: Basic Books, 1990.

36. Barker P. It's time to turn the tide. Nurs Times 1998;94:70.

37. Thyer B. The handbook of social work research methods: Los Angeles. Sage 2010.

38. Morgan DL, Morgan RK. Single-case research methods for the behavioral and health sciences. London: Sage, 2009.

39. Kazdin AE. Single-case research designs: methods for clinical and applied settings. New York: Oxford University Press, 2011.

40. Craig P, Dieppe P, Macintyre S, et al.Developing and evaluating complex interventions: the new Medical Research Council guidance. BMJ 2008;337:a1655. ARTN a1655.

41. Wyder M, Bland R, Blythe A, et al. Therapeutic relationships and involuntary treatment orders: service users' interactions with health-care professionals on the ward. Int $J$ Ment Health Nurs 2015;24:181-9

42. Swanson KM. Predicting depressive symptoms after miscarriage: a path analysis based on the Lazarus paradigm. J Womens Health Gend Based Med 2000;9:191-206.

43. Huskisson EC. MEASUREMENT OF PAIN. The Lancet 1974;304:1127-31.

44. Zigmond AS, Snaith RP. The hospital anxiety and depression scale. Acta Psychiatr Scand 1983;67:361-70.

45. Herrmann C. International experiences with the Hospital Anxiety and Depression Scale--a review of validation data and clinical results. $J$ Psychosom Res 1997;42:17-41.

46. Lisspers J, Nygren A, Söderman E. Hospital Anxiety and Depression Scale (HAD): some psychometric data for a Swedish sample. Acta Psychiatr Scand 1997;96:281-6.

47. Cohen S, Kamarck T, Mermelstein R. A global measure of perceived stress. J Health Soc Behav 1983;24:385-96.

48. Nordin M, Nordin S. Psychometric evaluation and normative data of the Swedish version of the 10 -item perceived stress scale. Scand J Psychol 2013;54:502-7.

49. Glasberg AL, Eriksson S, Dahlqvist V, et al. Development and initial validation of the Stress of Conscience Questionnaire. Nurs Ethics 2006;13:633-48.

50. Glasberg AL, Eriksson S, Norberg A. Burnout and 'stress of conscience' among healthcare personnel. J Adv Nurs 2007;57:392-403.

51. Schröder A, Larsson BW, Ahlström G. Quality in psychiatric care: an instrument evaluating patients' expectations and experiences. Int $J$ Health Care Qual Assur 2007;20:141-60.

52. Schröder A, Larsson BW, Ahlström G, et al. Psychometric properties of the instrument quality in psychiatric care and descriptions of quality of care among in-patients. Int J Health Care Qual Assur 2010;23:554-70.

53. Kvale S, Brinkmann S. InterViews: learning the craft of qualitative research interviewing. Los Angeles: Sage Publications, 2009.

54. Sandelowski M. Focus on qualitative method. Sample size in qualitative research. Res Nurs Health 1995;18:179-83.

55. De Chesnay M. Nursing research using ethnography: qualitative designs and methods in nursing. New York: Springer Publishing, 2014.

56. Richards DA, Hallberg R I. Complex interventions in health: an overview of research methods. Abingdon, Oxon: Routledge, 2015.

57. Graneheim UH, Lundman B. Qualitative content analysis in nursing research: concepts, procedures and measures to achieve trustworthiness. Nurse Educ Today 2004;24:105-12.

58. Hallberg IR. Moving nursing research forward towards a stronger impact on health care practice? Int J Nurs Stud 2009;46:407-12.

59. Moore GF, Audrey S, Barker M, et al. Process evaluation of complex interventions: Medical Research Council guidance. BMJ 2015;350:h1258.

60. Storm M, Edwards A. Models of user involvement in the mental health context: intentions and implementation challenges. Psychiatr Q 2013;84:313-27. 